\title{
The Phase Probability for Some Excited Binomial States
}

\section{Darwish}

Faculty of Education, Suez Canal University at Al-Arish, Egypt.

In this paper, the phase properties in Pegg-Barnett formalism are considered. The phase distribution is calculated and discussed for the excited binomial state, the even-excited binomial state and the odd-excited binomial state. 


\section{Introduction:}

Classically there are two theories for light: waves (Huygens) and corpuscular (Newton). Since the advent of quantum mechanics the concept of the photon had been introduced. Light consists of photons carrying energy quanta proportional to the frequency $E=\hbar \omega$, and momentum proportional to the wave number $p=\hbar c k$. The quantum description of the state of light depends on the quantum description of the photon. The state that describes $n$ photons is the Fock number state $|n\rangle$, which is an eigenstate of the photon number operator $\hat{n}=\hat{a}^{\dagger} \hat{a}$, i.e. $\hat{a}^{\dagger} \hat{a}|n\rangle=n|n\rangle$ where $\hat{a},(\hat{a}|n\rangle=\sqrt{n}|n-1\rangle)$ and $\hat{a}^{\dagger},\left(\hat{a}^{\dagger}|n\rangle=\sqrt{n+1}|n+1\rangle\right)$ are the annihilation and creation operators of one photon, respectively. However, another state was introduced by Glauber (1963) see for example $[1,2]$ namely the coherent state which describes a field with a fixed phase while the number of photons is not fixed. However, there is an average value for this photon number. The number of photons has a Poissonian distribution. This state (the coherent state) $|\alpha\rangle$, has the photon number distribution $P(n)=\frac{e^{-\bar{n}}}{n !} n^{\bar{n}}$, where $\bar{n}$ is the mean photon number $\bar{n}=|\alpha|^{2}, \alpha$ is a complex number, whose modulus is the amplitude of the field mode, and its phase is the phase of the state. This state describes very closely the laser field. Theoretical descriptions for other states followed.

A state was introduced which bridges the gab between the Fock state and the coherent state by taking mathematical limits. This state is the binomial state (BS) [3], where the probability distribution function for finding $\$ n \$$ photons is given by the binomial distribution,

$$
P(n)=\left\{\begin{array}{ll}
\frac{M !}{n !(M-n) !}|\eta|^{2 n}\left(1-|\eta|^{2}\right)^{M-n} & \text { for } \quad n \leq M \\
0 & \text { for } n>M
\end{array}\right\}
$$

A realization of such state can be thought of as a 2-level atom in its excited state that emits a photon to go down to its ground state, if the probability of detecting a photon is $|\eta|^{2}$ then failure to detect such photon is $\left(1-|\eta|^{2}\right)$. Hence the 
probability to detect $n$ counts out of $M$ experiments is given by equation (1). It is easy to see that as $\eta \rightarrow 0$ then $P(0)=1, P(n)=0, n \neq 0$, while when $\eta \rightarrow 1$ then $P(M)=1, P(n)=0, n \neq M$, while when $M \rightarrow \infty, \eta \rightarrow 0$ such that $M|\eta|^{2} \rightarrow \bar{n}$ a fixed number, we get $P(n)=\frac{e^{-\bar{n}}}{n !} n^{\bar{n}}$ the Poissonian distribution. The state that describes this situation is the BS [3] $|M, \eta\rangle$ given by

$$
|M, \eta\rangle=\sum_{n=0}^{M} \sqrt{\frac{M !}{n !(M-n) !}} \eta^{n}\left(1-|\eta|^{2}\right)^{\frac{M-n}{2}}|n\rangle
$$

Another state introduced to interpolate between the thermal and the coherent states, is the negative binomial state (NBS) [4-6]. As further example, the generalized geometric state interpolates between the number state and the (non-pure) chaotic state [7-9]. Furthermore, the even (odd) BS interpolates, between the even (odd) coherent state and the even (odd) number state [10,11]. It was reported that under suitable conditions, superposition of coherent states could be produced if a coherent state is allowed to propagate through an amplitude dispersive medium [12]. Thus quantum superposition of BS can be produced in the same way, since the BS tends to a coherent state as a limiting case. It would be interesting to refer to the excited binomial states (EBS) of the radiation field, which can be generated by repeated application of the photon creation operator on BS [13]. They reduce to Fock states and excited coherent states (ECS) in certain limits and can be viewed as intermediate states between Fock states and the ECS [13]. Also, the odd-excited binomial states (OEBS) and the even excited binomial states (EEBS) of the radiation field which are introduced by repeated application of the photon creation operator on EBS. These states interpolate between the odd (even) number state and the odd (even) displaced Fock state [14].

The aim of this work is to study the phase properties for both EBS, EEBS and OEBS in the Pegg-Barnett formalism $[15,16]$.

In section 2 the phase distribution function is introduced. In section 3 the phase distribution related to the EBS is calculated. Also, for the states of EEBS and OEBS the phase distributions function are calculated in sections 4 , 5. Finally conclusion is given in section 6 . 


\section{The phase distribution function:}

The notion of the phase in quantum optics has found renewed strong interest because of the existence of phase-dependent quantum noise. In this section, the phase properties using the Pegg-Barnett method $[15,16]$ are studied. This is based on the phase states $|\Theta\rangle$, which are defined as

$$
|\Theta\rangle=\frac{1}{\sqrt{s+1}} \sum_{n=0}^{s} \exp \left(i n \theta_{m}\right)|n\rangle,
$$

where

$$
\theta_{m}=\theta_{0}+\frac{2 \pi m}{s+1} ; m=0,1, \ldots, s
$$

The value of $\theta_{0}$ is arbitrary; it indicates a specific bases set of $(s+1)$ mutually orthogonal phase states. In fact the phase states $\left|\Theta_{m}\right\rangle$ are eigenstates of Hermitian phase operator $\hat{\Phi}_{\theta}$ given by

$$
\hat{\Phi}_{\theta}=\sum_{m=0}^{s} \theta_{m}\left|\Theta_{m}\right\rangle\left\langle\Theta_{m}\right|
$$

The state of the form

$$
|b\rangle=\sum_{m=0}^{s} b_{n} \exp (i n \Psi)|n\rangle
$$

is called a partial phase state [15], where $b_{n}$ are real and positive and $\Psi$ is a phase. From equations $(3,6)$, one can calculate the expectation value and the variance for the phase operator $\hat{\Phi}_{\theta}$ with respect to the partial phase state; we have

$$
\begin{aligned}
& \left\langle\hat{\Phi}_{\theta}\right\rangle=\Psi \\
& \left\langle\Delta \hat{\Phi}_{\theta}^{2}\right\rangle=\frac{\pi^{2}}{3}+4 \sum_{n>m} \sum_{m} \frac{(-1)^{(n-m)} b_{n} b_{m}}{(n-m)^{2}}
\end{aligned}
$$

The phase probability distribution for the partial phase state is given as

$$
P(\theta)=\left|\left\langle\Theta_{m} \mid b\right\rangle\right|^{2}
$$


Since the density of phase states is $\frac{s+1}{2 \pi}$, thus in the continuum limit $s \rightarrow \infty$, equation (9) reduces to

$$
P(\theta)=\frac{1}{2 \pi}\left[1+2 \sum_{n>m} \sum_{m} b_{n} b_{m} \cos [(n-m) \theta]\right]
$$

In what follows one calculate this function for different states.

\section{The phase distribution function of EBS:}

The EBS is defined as [13]

$$
|k, \eta, M\rangle=\lambda \sum_{n=0}^{M} C_{n}^{M}(k)|n+k\rangle
$$

with $\lambda$ the normalization constant given by

$$
|\lambda|^{-2}=\sum_{n=0}^{M} \frac{M !}{n !(M-n) !} \frac{(n+k) !}{n !} \eta^{2 n}\left(1-|\eta|^{2}\right)^{M-n}
$$

and

$$
C_{n}^{M}(k)=\sqrt{\frac{M !}{n !(M-n) !}} \eta^{2 n}\left(1-|\eta|^{2}\right)^{M-n}
$$

Here $n$ and $M$ are integers. $\eta$ is in general complex with $0 \leq|\eta| \leq 1$. By using equations. (10) and (11) the phase distribution function for this state is given by

$P(\theta)=\frac{1}{2 \pi}\left[\begin{array}{l}1+2|\lambda|^{2} \sum_{n=1}^{M} \sum_{m=0}^{n-1} \frac{M !}{n ! m !}\left(1-|\eta|^{2}\right)^{\left(\frac{M-n}{2}\right)}\left(1-|\eta|^{2}\right)^{\left(\frac{M-m}{2}\right)} \\ \sqrt{\frac{(n+k) !(m+k) !}{(M-n) !(M-m) !}} \eta^{n} \eta^{*^{m}} \cos [(n-m) \theta]\end{array}\right]$

In Fig. (1), $P(\theta)$ given by equation. (14) is plotted against the parameter $\eta$, for $M=5$ and $k=1$. In this figure we can see that there is only one stretching peak along the $\eta$ axis at $\theta=0$. At $\eta=0$, then $P(\theta)=\frac{1}{2 \pi}$, and the phase is 
lost. But at $\eta$ increases, the phase starts to build up, and the information about the phase can be attained. At $\eta$ become 1 , then $P(\theta)=\frac{1}{2 \pi}$.

From Fig. (2), we can see that as $k$ increases with constant $M$, then the maximum value of $P(\theta)$, at $\theta=0$ moves to lower values of $\eta$. Keeping $k$ constant and varying $M$, the maximum values of $P(\theta)$ at $\theta=0$ increases slightly as $M$ increase.

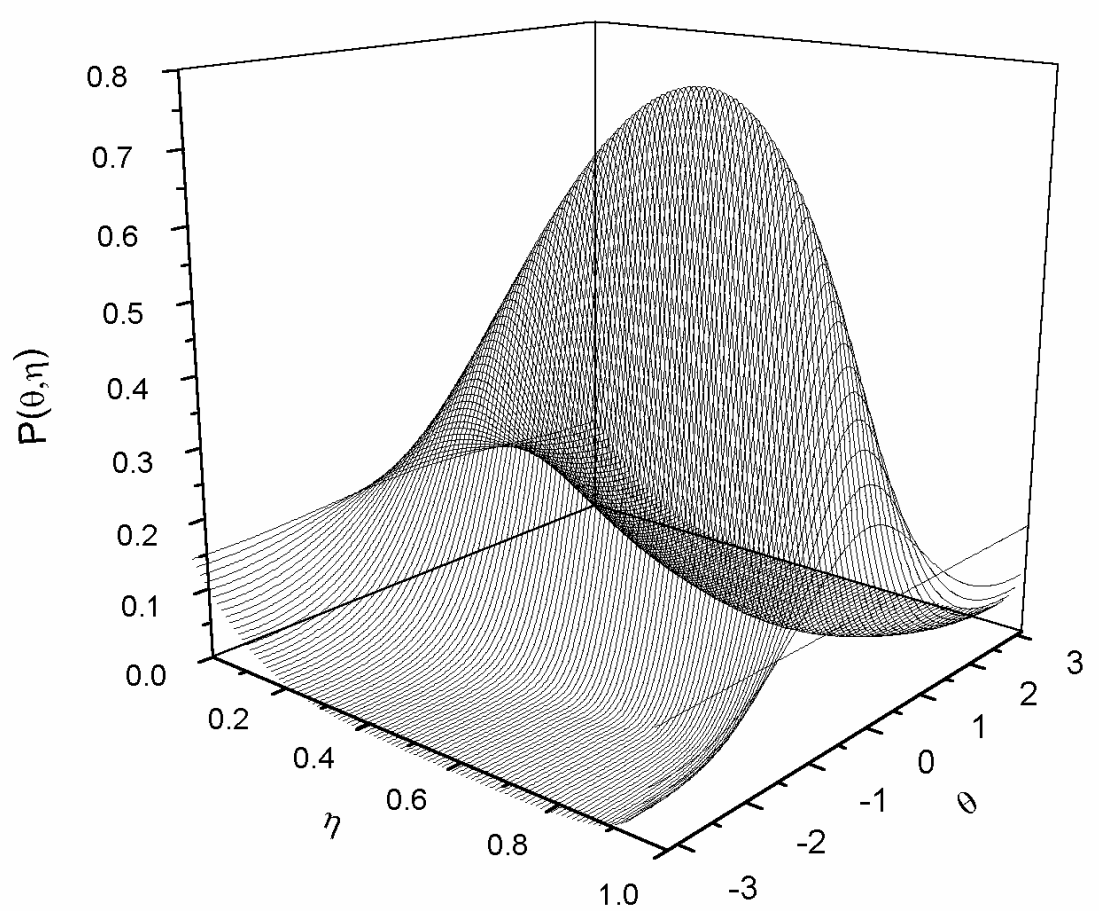

Fig. (1): The phase distribution for $E B S$ at $M=5, k=1$.

\section{The phase distribution function of EEBS:}

The EEBS is introduced through the following definition

$$
|k, \eta, M\rangle_{e}=\lambda^{\prime} \sum B_{n}^{M}(k)|n+k\rangle
$$

such that $|n+k\rangle$ are always even. There are the following cases

(i) even $k$ i.e. $k=2 k_{0}$ 


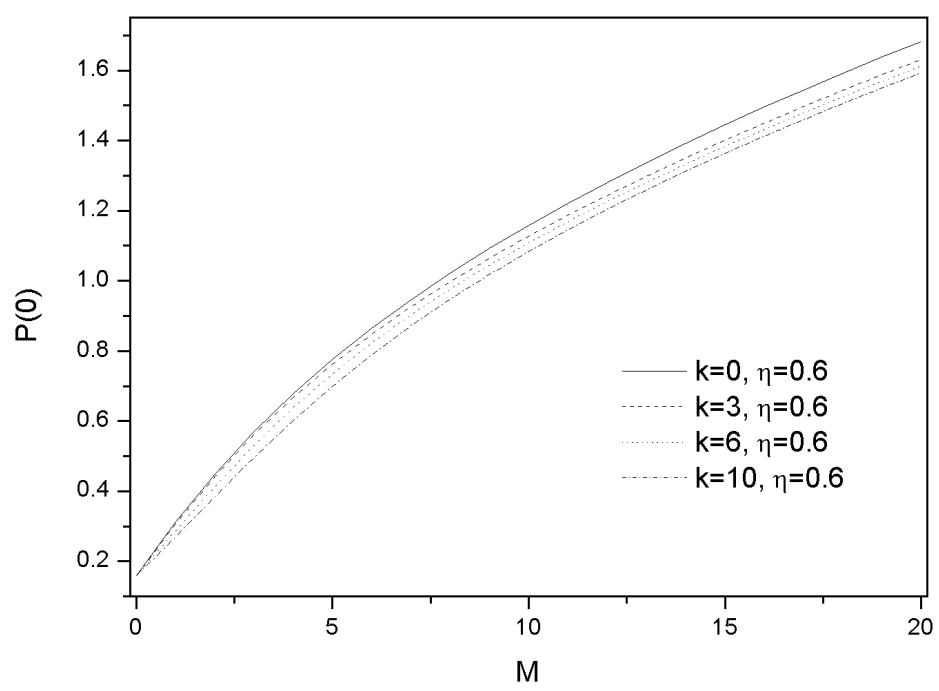

Fig. (2): The maximum values of $P(0)$ against parameter $M$.

$$
\left|2 k_{0}, \eta, M\right\rangle_{e}=\lambda_{e} \sum_{n=0}^{\left[\frac{M}{2}\right]} B_{2 n}^{M}\left(2 k_{0}\right)\left|2 n+2 k_{0}\right\rangle
$$

where

$$
B_{2 n}^{M}\left(2 k_{0}\right)=\sqrt{\frac{M !}{(2 n) !(M-2 n) !}} \eta^{2 n}\left(1-|\eta|^{2}\right)^{\left.\frac{M-2 n}{2}\right)} \sqrt{\frac{\left(2 n+2 k_{0}\right) !}{(2 n) !}}
$$

With $\left[\frac{M}{2}\right]$ being the largest integer less than or equal to $\frac{M}{2} \cdot \lambda_{e}$ is the normalization constants of EEBS for $k$ even. $B_{2 n}^{M}$ is the probability amplitudes of even excited binomial distribution. The normalization constant of this state can be written as

$$
\left|\lambda_{e}\right|^{-2}=\sum_{n=0}^{\left[\frac{M}{2}\right]} \frac{M !}{(2 n) !(M-2 n) !} \frac{\left(2 n+2 k_{0}\right) !}{(2 n) !} \eta^{4 n}\left(1-|\eta|^{2}\right)^{(M-2 n)}
$$

By applying equations (10) and (15), the phase distribution function for this state is given by 


$$
P(\theta)=\frac{1}{2 \pi}\left[\begin{array}{l}
1+2\left|\lambda_{e}\right|^{2} \sum_{n=1}^{\left[\frac{M}{2}\right]} \sum_{m=0}^{n-1} \frac{M !}{(2 n) !(2 m) !}\left(1-|\eta|^{2}\right)^{\left(\frac{M-2 n}{2}\right)}\left(1-|\eta|^{2}\right)^{\left(\frac{M-2 m}{2}\right)} \\
\sqrt{\frac{\left(2 n+2 k_{0}\right) !\left(2 m+2 k_{0}\right) !}{(M-2 n) !(M-2 m) !} \eta^{2 n} \eta^{* 2 m} \cos [(2 n-2 m) \theta]}
\end{array}\right]
$$

(ii) odd $k$ i.e. $k=2 k_{1}+1$

$$
\begin{gathered}
\left|2 k_{1}+1, \eta, M\right\rangle_{e}=\lambda_{e}^{\prime} \sum_{s=0}^{\left[\frac{M+1}{2}\right]} B_{2 s-1}^{M}\left(2 k_{1}+1\right)\left|2 s+2 k_{1}\right\rangle \\
B_{2 s-1}^{M}\left(2 k_{1}+1\right)=\sqrt{\frac{M !}{(2 s-1) !(M-2 s+1) !}} \eta^{2 s-1}\left(1-|\eta|^{2}\right)^{\left(\frac{M-2 s+1}{2}\right)} \sqrt{\frac{\left(2 s+2 k_{1}\right) !}{(2 s-1) !}}
\end{gathered}
$$

$\lambda_{e}^{\prime}$ is the normalization constants of EEBS for $k$ odd. Also $B_{2 s-1}^{M}$ is the probability amplitudes of even excited binomial distribution. The normalization constant of this case is given by

$$
\left|\lambda_{e}^{\prime}\right|^{-2}=\sum_{s=1}^{\left[\frac{M+1}{2}\right]} \frac{M !}{(2 s-1) !(M-2 s+1) !} \frac{\left(2 s+2 k_{1}\right) !}{(2 s-1) !} \eta^{4 s-2}\left(1-|\eta|^{2}\right)^{(M-2 s+1)}
$$

By applying equations (10) and (20), the phase distribution function for this state reads,

$$
P(\theta)=\frac{1}{2 \pi}\left[\begin{array}{l}
1+2\left|\lambda_{e}^{\prime}\right|^{\left[\frac{M+1}{2} \sum_{s=2}^{2}\right.} \sum_{s^{\prime}=1}^{s-1} \frac{M !}{(2 s-1) !\left(2 s^{\prime}-1\right) !}\left(1-|\eta|^{2}\right)^{\left(\frac{M-2 s+1}{2}\right)}\left(1-|\eta|^{2}\right)^{\left.\frac{M-2 s^{\prime}+1}{2}\right)} \\
\sqrt{\frac{\left(2 s+2 k_{1}\right) !\left(2 s^{\prime}+2 k_{1}\right) !}{(M-2 s+1) !\left(M-2 s^{\prime}+1\right) !}} \eta^{(2 s-1)} \eta^{*\left(2 s^{\prime}-1\right)} \cos \left[\left(2 s-2 s^{\prime}\right) \theta\right]
\end{array}\right]
$$

In Fig. (3), $P(\theta)$ given by equations $(19,23)$ is plotted against the parameter $\eta$, for $M=8$ and $k=2$. We can see that there are two peaks along the $\eta$ axis at $\theta=0$ and $\pi$. Calculations of the effect of the values of $M$ and $k$ on the maximum values of $P(\theta)$ are summarized. In general, the same trend is noticed as the earlier case of the EBS. 


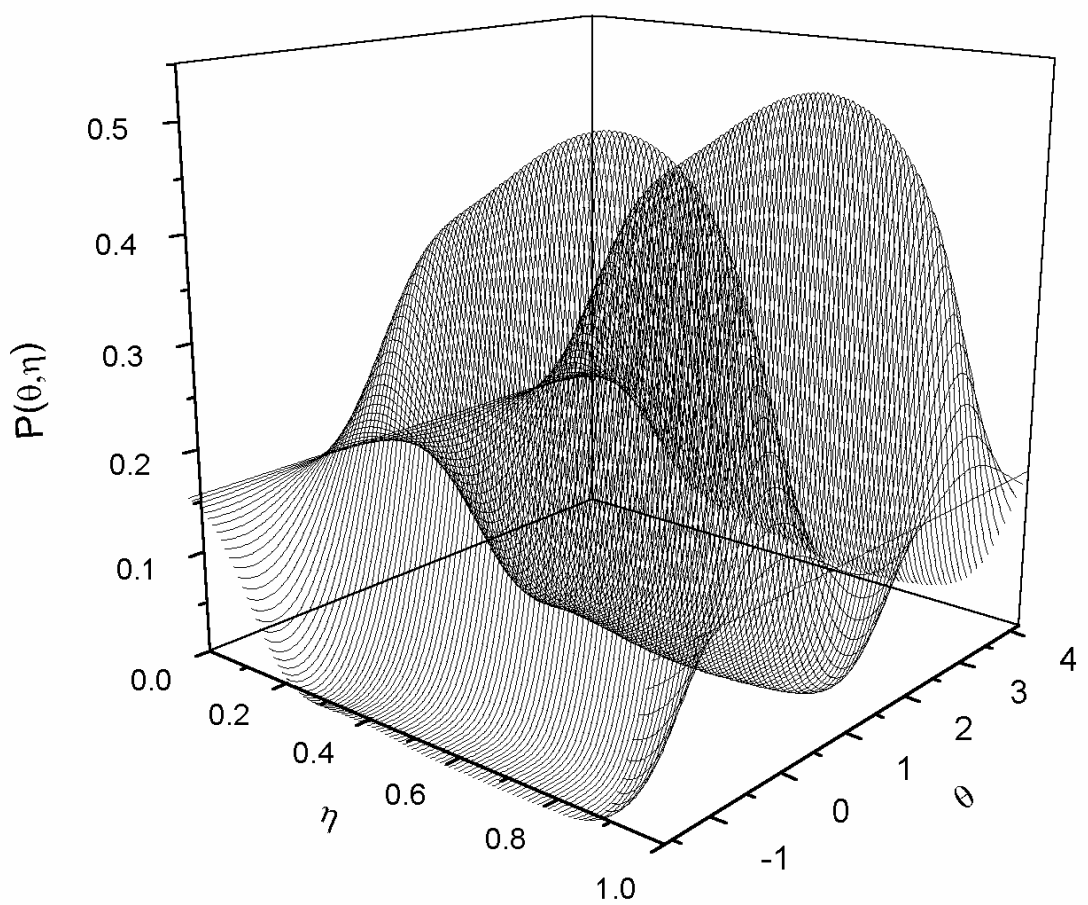

Fig. (3): The phase distribution for EEBS at $M=8, k=2$.

\section{The phase distribution function of OEBS:}

The OEBS can be introduced through the following definition

$$
|k, \eta, M\rangle_{o}=\lambda^{\prime} \sum C_{n}^{M}(k)|n+k\rangle
$$

such that $|n+k\rangle$ are always odd [14]. Similarly, we have the following two cases(i) even $k$ i.e. $k=2 k_{0}$

$$
\left|2 k_{0}, \eta, M\right\rangle_{o}=\lambda \sum_{n=0}^{\left[\frac{M-1}{2}\right]} C_{2 n+1}^{M}\left(2 k_{0}\right)\left|2 n+2 k_{0}+1\right\rangle
$$

where

$$
C_{2 n+1}^{M}\left(2 k_{0}\right)=\sqrt{\frac{M !}{(2 n+1) !(M-2 n-1) !}} \eta^{(2 n+1)}\left(1-|\eta|^{2}\right)^{\left(\frac{M-2 n-1}{2}\right)} \sqrt{\frac{\left(2 n+2 k_{0}+1\right) !}{(2 n+1) !}}
$$

$\lambda_{o}$ is the normalization constant of OEBS for $k$ even, given by 


$$
\left|\lambda_{o}\right|^{-2}=\sum_{n=0}^{\left[\frac{M-1}{2}\right]} \frac{M !}{(2 n+1) !(M-2 n-1) !} \frac{\left(2 n+2 k_{0}+1\right) !}{(2 n+1) !} \eta^{(4 n+2)}\left(1-|\eta|^{2}\right)^{(M-2 n-1)}
$$

By using equations (10) and (25), the phase distribution function for this state is given by

$$
P(\theta)=\frac{1}{2 \pi}\left[\begin{array}{l}
1+2\left|\lambda_{o}\right|^{2} \sum_{n=1}^{\left.\frac{M-1}{2}\right]} \sum_{m=0}^{n-1} \frac{M !}{(2 n+1) !(2 m+1) !}\left(1-|\eta|^{2}\right)^{\left(\frac{M-2 n-1}{2}\right)}\left(1-|\eta|^{2}\right)^{\frac{\left(\frac{M-2 m-1}{2}\right)}{(M-2 n-1) !(M-2 m-1) !} \eta^{(2 n+1)} \eta^{*(2 m+) 1} \cos [(2 n-2 m) \theta]} \\
\sqrt{\frac{\left(2 n+2 k_{0}+1\right) !\left(2 m+2 k_{0}+1\right)}{(M-2 m-1}}
\end{array}\right]
$$

(ii) odd $k$ i.e. $k=2 k_{1}+1$

$$
\left|2 k_{1}+1, \eta, M\right\rangle_{o}=\lambda_{o}^{\prime} \sum_{s=0}^{\left[\frac{M}{2}\right]} C_{2 s}^{M}\left(2 k_{1}+1\right)\left|2 s+2 k_{1}+1\right\rangle
$$

where

$$
C_{2 s}^{M}\left(2 k_{1}+1\right)=\sqrt{\frac{M !}{(2 s) !(M-2 s) !}} \eta^{2 s}\left(1-|\eta|^{2}\right)^{\left.\frac{M-2 s}{2}\right)} \sqrt{\frac{\left(2 s+2 k_{1}+1\right) !}{(2 s) !}}
$$

$\lambda_{e}^{\prime}$ is the normalization constants of OEBS for $k$ odd, given by

$$
\left|\lambda_{o}^{\prime}\right|^{-2}=\sum_{s=1}^{\left[\frac{M}{2}\right]} \frac{M !}{(2 s) !(M-2 s) !} \frac{\left(2 s+2 k_{1}+1\right) !}{(2 s) !} \eta^{4 s}\left(1-|\eta|^{2}\right)^{(M-2 s)}
$$

By using equations (10) and (29), the phase distribution function for this state is given by

$$
P(\theta)=\frac{1}{2 \pi}\left[\begin{array}{l}
1+2\left|\lambda_{o}^{\prime}\right|^{2} \sum_{s=1}^{\left[\frac{M}{2}\right]} \sum_{s^{\prime}=0}^{s-1} \frac{M !}{(2 s) !\left(2 s^{\prime}\right) !}\left(1-|\eta|^{2}\right)^{\left(\frac{M-2 s}{2}\right)}\left(1-|\eta|^{2}\right)^{\left(\frac{M-2 s^{\prime}}{2}\right)} \\
\sqrt{\frac{\left(2 s+2 k_{1}+1\right) !\left(2 s^{\prime}+2 k_{1}+1\right) !}{(M-2 s) !\left(M-2 s^{\prime}\right) !}} \eta^{(2 s)} \eta^{*\left(2 s^{\prime}\right)} \cos \left[\left(2 s-2 s^{\prime}\right) \theta\right]
\end{array}\right]
$$

In Fig. (4), $P(\theta)$ given by equations $(28,32)$ is plotted against the parameter $\eta$, for $M=15$ and $k=1$. Again, we see that there are two stretching peaks along the $\eta$ axis at $\theta=0$ and $\pi$. The effect of the values of $M$ and $k$ on the maximum values of $P(\theta)$ is the same with the cases of EBS and EEBS. 


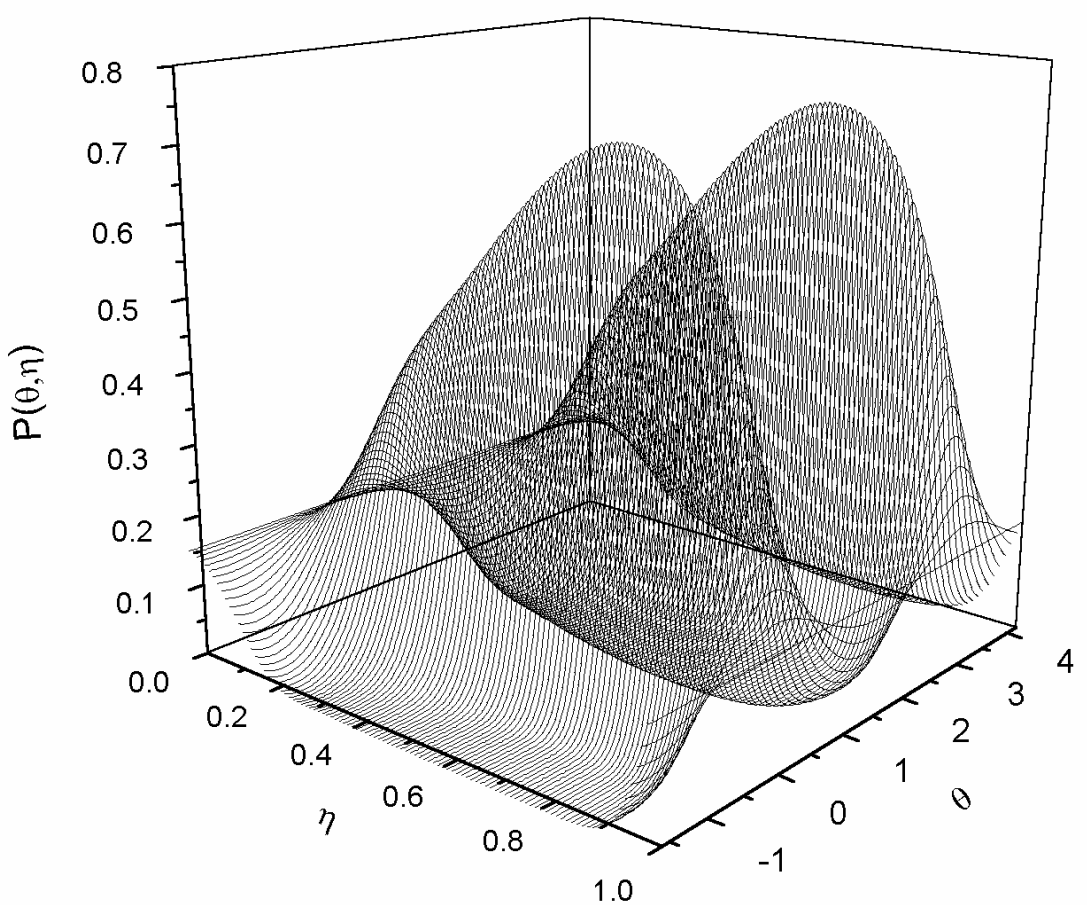

Fig. (4): The phase distribution for OEBS at $M=15, k=1$.

\section{Conclusion:}

In this article, the phase distribution function in the sense of PeggBarnett formalism has been calculated and plotted for the cases of the EBS, EEBS and the OEBS. This investigation shows a loss of phase information $\left(P(\theta)=\frac{1}{2 \pi}\right)$ as $\eta \rightarrow 0$ or $\eta \rightarrow 1$ which reflects the fact of having a Fock state. But for $0<\eta<1$ peak accurse around $\theta=0$, for the case of EBS, and two peaks at $\theta=0$ and $\pm \pi$, for the cases of EEBS and OEBS which means that the phase is built up by adding more Fock states. This means that these states are partially coherent phase states. 


\section{References:}

1. Glauber R., J. Phys. Rev. B1, 2766 (1963).

2. Perina, J., Quantum statistics of linear and nonlinear optical phenomena, Reidel, Dordrecht (1984), 78. And Walls, D. F. and Milburn, G., Quantum Optics, Springer Verlag Berlin (1994).

3. Stoler, D., Saleh, B. E. A. and Teich, M. C., Optica Acta, 32, 345 (1985).

4. Joshi, A. and Lawande, S. V., Optics Communications, 70, 21 (1989),.

5. Joshi, A. and Lawande, S. V., J. Mod. Opt., 38, 2009 (1991).

6. Agarwal, G. S., Physical Review A, 45, 1787 (1992).

7. Obada, A.-S.F., Hassan, S. S., Puri, R. R., and Abdalla, M. S., Phys. Rev. A, 48, 3174 (1993).

8. Batarfi, H. A., Abdalla, M. S., Obada, A.-S.F. and Hassan, S. S., Phys. Rev. A, 51, 2644 (1995).

9. Obada, A.-S.F., Yassin, O. M. and Barnett, S. M., J. Mod. Opt,. 44, 149 (1997).

10. Abdalla, M. S., Mahran, M. H. and Obada, A.-S.F., J. Mod. Opt., 41, 1889 (1994).

11. Obada, A.-S.F., Mahran, M. H., El-Orany, F. A. A. and Abdalla, M. S., Int. J. Theor. Phys., 35, 139 (1996).

12. Gerry, C. C., Optics Communication, 91, 247 (1992).

13. Wang, X. G., Fu, H. C., Int. J. Theor. Phys. 39, 1437 (2000),.

14. A. -S. F. Obada, M. Darwish, and H. H. Salah, Int. J. Theor. Phys. 41, 1755 (2002),

15. Barnett, S. M., Pegg, D. T., J. Mod. Opt., 36, 7 (1989).

16. Pegg, D. T., Barnett, S. M., Phys. Rev. A, 39, 1665 (1989). 